\title{
JD7 -Astrophysical Outflows and Associated Accretion Phenomena
}

\author{
Elisabete M. de Gouveia Dal Pino ${ }^{1}$ and Alex C. Raga ${ }^{2}$ \\ ${ }^{1}$ Universidade de São Paulo,Cidade Universitria, BR São Paulo, SP 05508-900, Brazil \\ ${ }^{2}$ Instituto de Ciencias Nucleares, Universidad Nacional Autónoma de México, México
}

\section{Preface}

Highly collimated supersonic jets and outflows are very frequent in several astrophysical environments. They are seen in young stellar objects (YSOs), proto-planetary nebulae, compact objects (like galactic black holes or microquasars, and X-ray binary stars), active galactic nuclei, and are also possibly associated to gamma-ray bursts (GRBs) and to ultra-high energy cosmic rays sources (UHECRs). Despite their different physical scales, all these outflow classes have strong morphological similarities, but questions such as what physics do they share? - or - can we find a universal mechanism of acceleration and collimation that operates in all classes? - remain matters of debate. The most accepted mechanism for their origin relies on a rotating accretion disk threaded by perpendicular large-scale magnetic fields and, though most of the systems producing jets contain an accretion disk around the central source, the real role that rotation and magnetic fields play in these processes is still not fully understood, nor are the highly non-linear physical processes connected to these jet-disk systems in the large parameter space involved.

Recent years have been marked by reviving worldwide interest in the studies of magnetic disk accretion and outflows both because of their potential relation to several astrophysical phenomena that are not well understood yet like GRBs and UHECRs, and also because of the fast advance in multi-dimensional computational modelling of these systems. Also, the large improvement in the observations with the building of new instrumentation and techniques now allow to resolve the base of some outflows very near the sources. The combination of multi-wavelength data has also given new insights into outflow phenomena. We thus felt that the time was ripe to revive the discussion of the origin of the astrophysical jets and their effects on the astrophysical environments. Particularly, holding it during the IAU General Assembly in Rio de Janeiro has attracted worldwide experts from a broad range of research fields.

The main scientific goal of this one-and-a-half-day Joint Discussion on Astrophysical Outflows and Associated Accretion Phenomena was to focus on understanding the driving mechanisms of jets from proto-stars (including their possible crucial link with star formation) to microquasars and AGNs. We have tried to emphasize their differences/similarities; the basic physics of the accretion-jet process in magnetized disks, including the transport of angular momentum and the development of reconnection and turbulent dynamo; cooling/heating processes, instabilities, shock structures and particle acceleration mechanisms in the jets; the impact of the jets on energy balance and turbulence feeding in the astrophysical environments; and the potential association of jet-accretion phenomena with GRBs and UHECRs.

The strategy was to try to create a powerful interdisciplinary synergy between the observational, theoretical and computational modelling domains of expertise. We had more than 110 participants from 28 countries, out of which 17 gave invited talks, 4 oral 
contributions and 88 poster presentations. All the sessions ended with discussion panels of the related topics and poster contributions. The meeting was concluded with a Summary Panel that highlighted what we considered the most relevant questions for continuing study. These open questions can be summarized as follows:

- To what extent are the magnetic fields advected from the environment and/or generated continuously inside the accretion disk through a dynamo process?

- What is the role of turbulence on angular momentum transport, dynamo action and large scale magnetic field generation within accretion disks?

- What is the Prandtl number regime in accretion disks over different scales (from YSO to relativistic sources)?

- What is the role of magnetic reconnection and coronal magnetic activity in the disk/coronal/jet launching process?

- Is there a connection between QPOs, general jet variability, and ejection phenomena with magnetic reconnection in the inner disk/corona boundary?

- What is the nature of the coupling between source magnetosphere and disk/corona magnetic field lines?

- Is jet collimation/recollimation of magnetic nature only?

- How relevant is steady jet production modelling?

- What is the Jet/accretion disk and star formation connection from low to high mass stars?

- What is the origin of molecular jets (prompt entrainment or intrinsic)?

- What will the new generation instruments (SKA, ALMA, etc.) be able to elucidate about the jet launching mechanism, rotation, etc?

We hope that we might be able to answer many of these questions in the near future.

Elisabete M. de Gouveia Dal Pino and Alex C. Raga, co-chairs of the SOC of the Joint Discussion on Astrophysical Outflows and Associated Accretion Phenomena, Rio de Janeiro, Brazil, August 2009 